\title{
Evaluation of Power Heat Losses in Multidomain Iron Particles Under the Influence of AC Magnetic Field in RF Range
}

\author{
Andrzej Skumiel • Milena Kaczmarek-Klinowska • \\ Milan Timko • Matus Molcan • Michał Rajnak
}

Received: 9 April 2012 / Accepted: 11 December 2012 / Published online: 4 January 2013

(C) The Author(s) 2012. This article is published with open access at Springerlink.com

\begin{abstract}
The magnetic properties and hyperthermia effect were studied in a magnetorheological fluid (MRF) containing iron particles of $1 \mu \mathrm{m}$ to $5 \mu \mathrm{m}$ in diameter. The measurements showed that the magnetization in the saturation state reaches a value of $171 \mathrm{~A} \cdot \mathrm{m}^{2} \cdot \mathrm{kg}^{-1}$ with very small values of coercivity and remanence. They also showed the ferromagnetic behavior in the system together with a value of the magnetic susceptibility of 1.7. Theoretical and experimental results of the calorimetric effect investigation under a changeable magnetic field of high frequency $(f=504 \mathrm{kHz})$ in an MRF will be presented in the article. The sample was subjected to an alternating magnetic field of different strengths $\left(H=0\right.$ to $\left.4 \mathrm{kA} \cdot \mathrm{m}^{-1}\right)$. It results from a theoretical analysis that the heat power density (released in the MRF sample) referenced to the eddy current is proportional to the square of frequency, the magnetic field amplitude, and the iron grain diameter. Experimental results indicate that there are some reasons for the released heat energy such as: energy losses from magnetic hysteresis and eddy currents induced in the iron grains. If the magnetic field intensity amplitude grows, the participation of losses connected with magnetic hysteresis is increased. From the calorimetric measurements, the conclusion is as follows: for a magnetic field $H<1946 \mathrm{~A} \cdot \mathrm{m}^{-1}$, the eddy current processes dominate in the heat generation mechanism, whereas hysteresis processes for the total release of thermal energy dominate for higher magnetic fields. Both mechanisms take equal parts in heating the tested sample at a magnetic field intensity amplitude $H=1946 \mathrm{~A} \cdot \mathrm{m}^{-1}$. The specific
\end{abstract}

\footnotetext{
A. Skumiel $(\varangle) \cdot$ M. Kaczmarek-Klinowska

Institute of Acoustics, Faculty of Physics, Adam Mickiewicz University, Umultowska 85,

61-614 Poznan, Poland

e-mail: skumiel@amu.edu.pl
}

M. Timko · M. Molcan · M. Rajnak

Institute of Experimental Physics, Department of Magnetism, Slovak Academy of Sciences,

Watsonova 47, 04001 Kosice, Slovakia 
absorption rate referenced to the mass unit of the MRF sample at the amplitude of the magnetic field strength $4 \mathrm{kA} \cdot \mathrm{m}^{-1}$ equals $24.94 \mathrm{~W} \cdot \mathrm{kg}^{-1}$ at a frequency $f=504 \mathrm{kHz}$.

Keywords Eddy current losses · Heating effect · Hysteresis losses ·

Magnetorheological fluid $\cdot$ Specific absorption rate

\section{Introduction}

The magnetorheological fluid (MRF) consists of ferro- or ferri-magnetic particles on the order of micron size dispersed in a viscous oil. These particles are multimagnetic-domain grains and they have no permanent magnetic moment [1]. There is no mutual magnetic attractive force between the particles in the MRF without an external magnetic field, and no particle coagulation occurs without an applied external magnetic field. The aim of the research is to estimate which mechanism is responsible for heating iron multidomain particles in an alternating RF magnetic field. Experimental results indicate that there are some reasons for released heat energy such as: energy losses from magnetic hysteresis and eddy currents induced in the iron grains. Simultaneously, in view of a large grain diameter size (circa $1 \mu \mathrm{m}$ to $5 \mu \mathrm{m}$ ), the energy loss caused by magnetic relaxation does not appear. It results from a theoretical analysis that the heat power density released in the MRF sample referenced to the eddy current is proportional to the square of frequency, the magnetic field amplitude, and the iron grain diameter. According to our theoretical analysis (see Appendix, Eq. 24), the effective power loss in the mass unit of the sphere, caused by eddy currents induced in the grains,

$$
P_{\mathrm{o}}=\frac{\left(\pi \mu_{\mathrm{o}} R f H\right)^{2}}{5 \rho_{\mathrm{Fe}} \rho},
$$

where $R$ is the radius of an iron grain, $f$ is the magnetic field frequency, $\rho$ is the the iron electrical resistivity, and $\rho_{\mathrm{Fe}}$ is the iron density.

In the case of a polydispersion system where the mean square of the grain diameter is $\left\langle d^{2}\right\rangle$, the effective power loss $\left\langle P_{\mathrm{o}}\right\rangle$ released, in mass units, equals

$$
\left\langle P_{\mathrm{o}}\right\rangle=\frac{\left(\pi \mu_{\mathrm{o}} f H\right)^{2}}{20 \rho_{\mathrm{Fe}} \rho}\left\langle d^{2}\right\rangle .
$$

The mean square of the grain diameter $\left\langle d^{2}\right\rangle$ may be calculated from the following expression:

$$
\left\langle d^{2}\right\rangle=d_{\mathrm{o}}^{2} \cdot \exp \left(2 \cdot \beta^{2}\right)
$$

where $d_{\mathrm{o}}$ and $\beta$ are parameters of the log-normal function.

The second mechanism responsible for iron multidomain particle heating in an alternating RF magnetic field is energy loss from magnetic hysteresis. Hysteresis losses are mainly due to the domain wall motion [2,3], and their value is given by the 

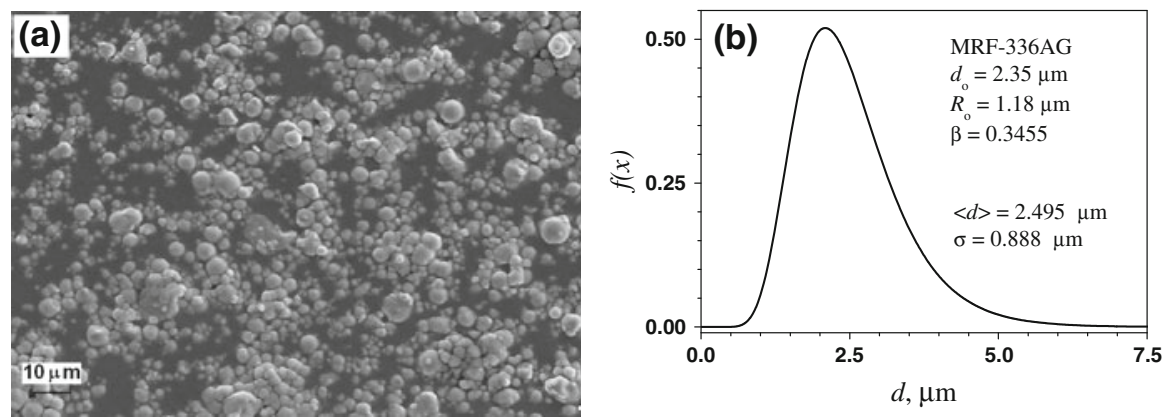

Fig. 1 (a) Appearance of MRF iron particles with the help of the scanning electron microscope and (b) log-normal function of the particle sizes

area of the hysteresis loop in an applied AC field. The coercivity $H_{\mathrm{c}}$ is usually strongly size dependent [4]. In this case, the power loss density depends on coercivity, and it is proportional to the frequency as is shown in the following expression:

$$
P=f \mu_{\mathrm{o}} \oint M \mathrm{~d} H,
$$

where $\mu_{\mathrm{o}}=4 \pi \times 10^{-7} \mathrm{H} \cdot \mathrm{m}^{-1}$ is the permeability of free space, $M$ is the magnetization, and $H$ is the magnetic field strength amplitude.

From Refs. [5-8], it follows that for particle systems with ferromagnetic behavior (i.e., hysteresis), a power of three is found at low field amplitudes (for Rayleigh losses). Thus, hysteresis losses, for so-called Rayleigh loops, may be well described by a thirdorder power law. In that case, it can be written as $P \propto H^{3}$. The authors selected this kind of medium for the experimental investigation because of the larger diameter sizes of iron magnetic grains ( $1 \mu \mathrm{m}$ to $5 \mu \mathrm{m})$; the presence of energy losses for eddy currents is larger than in nanoparticles. Figure 1a presents an image of iron particles which are the constituents of the MRF with help of the scanning electron microscope. In turn, in Fig. 1b, the course of a log-normal function of the size of the iron particles is shown. The log-normal function is described by two parameters: $d_{\mathrm{o}}=2.35 \mu \mathrm{m}$ and $\beta=0.3455$. The obtained mean magnetic diameter $\langle d\rangle=2.495 \mu \mathrm{m}$, and the standard deviation of particle size $\sigma=0.888 \mu \mathrm{m}$. The mean square of the grain diameter $\left\langle d^{2}\right\rangle=7.01 \cdot 10^{-12} \mathrm{~m}^{2}$.

MRFs are quite often used in industry for construction of vibration dampers. As we can find out from a leaflet producer, the MR fluids are suspensions of micron-sized, magnetic particles in silicone oil. This carrier liquid is a perfect electrical insulator in practice.

\section{Experimental Methods}

\subsection{Magnetic Properties}

The magnetic measurements were carried out by a SQUID magnetometer of Quantum Design in an external magnetic field up to $3 \mathrm{~T}$ at $293 \mathrm{~K}$. The hysteresis loop of the 
Fig. 2 Magnetization curve of the studied magnetorheological fluid at $293 \mathrm{~K}$

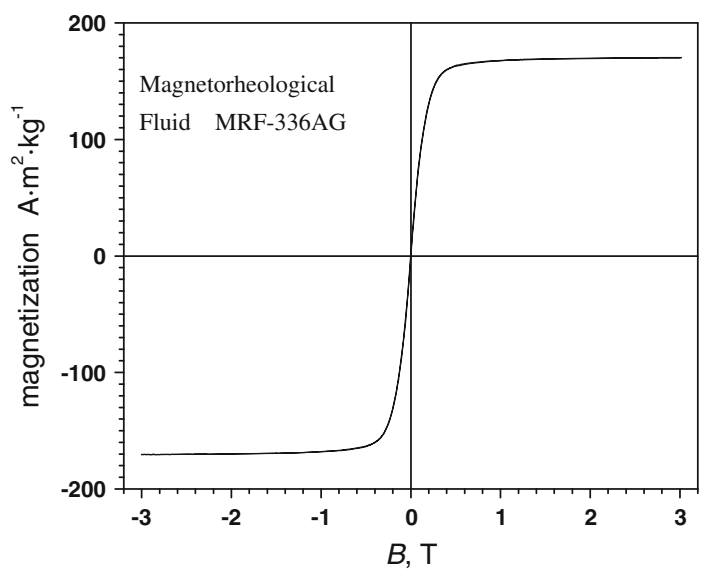

magnetorheological fluid MRF-336AG is presented in Fig. 2. The measured magnetization in the saturation state is $173 \mathrm{~A} \cdot \mathrm{m}^{2} \cdot \mathrm{kg}^{-1}$. This value is smaller than the value for bulk pure Fe reported in the literature as $213 \mathrm{~A} \cdot \mathrm{m}^{2} \cdot \mathrm{kg}^{-1}$ [9], and it corresponds to a magnetic volume concentration of $81.2 \%$ assuming that the saturation magnetization for bulk pure $\mathrm{Fe}$ is $213 \mathrm{~A} \cdot \mathrm{m}^{2} \cdot \mathrm{kg}^{-1}$. The remanent magnetization is $3.9 \mathrm{~A} \cdot \mathrm{m}^{2} \cdot \mathrm{kg}^{-1}$.

The measured coercivity was $1.81 \mathrm{mT}$ which means that the value was higher than for the bulk material $(0.101 \mathrm{mT})$. Some possible reasons for the higher coercivity of iron particles are the presence of impurities, defects, and an oxide layer on the iron particles. It is known that such defects can cause domain pinning, and thereby increasing the coercivity.

Measurements of the dynamic properties of the magnetorheological sample were carried out by AC Susceptometer DYNOMAG of Imego AB. At all frequencies measured in the range up to $10^{5} \mathrm{~Hz}$, a constant value of the real part susceptibility of 1.7 was observed. It maybe meant that no agglomeration of particles takes place, which can support the fact of the small value of the coercivity.

\subsection{Calorimetric Experiments}

Calorimetric investigations were performed in the testing system presented in Fig. 3. The tested medium was MagnetoRheological Fluid MRF-336AG produced by LORD Corporation. Two vials with an MR fluid and with silicone oil were placed in the air gap $(l=0.01 \mathrm{~m})$ of the magnetic system composed of ferrite with a transverse intersection of $0.0018 \mathrm{~m}^{2}$. To the magnetic ferrite core coil with 12 turns of copper winding (inductance $L_{\mathrm{o}}=100 \mu \mathrm{H}$ ), there was connected in series a capacitive decade to the power amplifier (AL-300-HF-A) with $300 \mathrm{~W}$ power. The capacitive decade is used in order to compensate for the inductive reactance.

For the condition of series voltage resonance, both (inductive and capacitive) reactances diminish and the current reaches its maximum value. Then the magnetic field also attains its maximum value. One turn of winding was applied to be observed and 


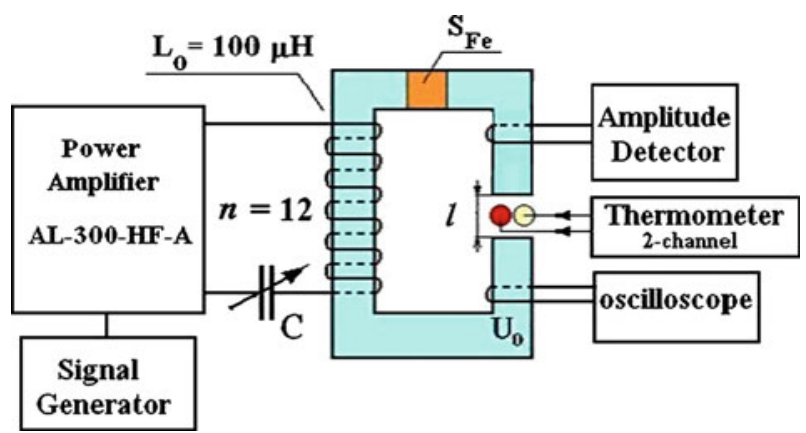

Fig. 3 Schematic diagram of experimental setup for measuring hyperthermal effect

to monitor the time path of the voltage which is added to the magnetic ferrite core. The second winding was used for amplitude detection and induced voltage amplitude measurement. This voltage is proportional to a magnetic flux value flowing in the magnetic system. It can be proved that the magnetic field intensity amplitude $H_{\mathrm{o}}$ in the air gap equals

$$
H_{\mathrm{o}}=\frac{U_{\mathrm{o}}}{2 \pi f S_{\mathrm{Fe}} \mu_{\mathrm{o}}},
$$

where $U_{\mathrm{o}}$ is the voltage amplitude induced through the magnetic flux in one turn of winding placed in the air gap, and $S_{\mathrm{Fe}}$ is the transverse intersection surface of the ferrite.

The same winding placed on the magnetic ferrite core indicates more flux than in the air gap because magnetic flux scattering appears in the vicinity of the gap. The temperature was measured in a differential system with the help of a thermometer with two fiber optic temperature sensors produced by FISO Technologies Inc. The measurement uncertainty of the magnetic strength amplitude is equal to $30 \mathrm{~A} \cdot \mathrm{m}^{-1}$, whereas the uncertainty of the temperature is equal to $0.1 \mathrm{~K}$.

The measurements were carried out at a frequency of $f=504 \mathrm{kHz}$ for some selected magnetic field intensity values.

In Fig. 4, time plots of the temperature difference between the vials with different MR values of the magnetic strength amplitude are presented. The magnetic field was switched on in $30 \mathrm{~s}$.

\section{Analysis}

For the experimental data (in the $30 \mathrm{~s}$ to $150 \mathrm{~s}$ time range), the exponential function was fitted in the following form:

$$
T(t)=\Delta T\left(1-\exp ^{\left(-\frac{t}{\tau}\right)}\right)
$$

where $\Delta T$ is the difference in temperature between two vials with MR fluids and with silicone oil at a steady state (at $t \longrightarrow \infty$ ), and $\tau$ is the time constant in the heating process.

The initial value of the expression $(\mathrm{d} T / \mathrm{d} t)_{t=0}=\Delta T / \tau[10]$. 
Fig. 4 Time courses of the temperature difference between the vials with MR fluids and with silicone oil during RF heating
Fig. 5 Dependence of $(\mathrm{d} T / \mathrm{d} t)_{t=0}$ on the alternating magnetic field strength amplitude $H$ at a frequency $f=504 \mathrm{kHz}$ for MRF-336AG sample. Their constituents were derived from eddy currents and hysteresis phenomena
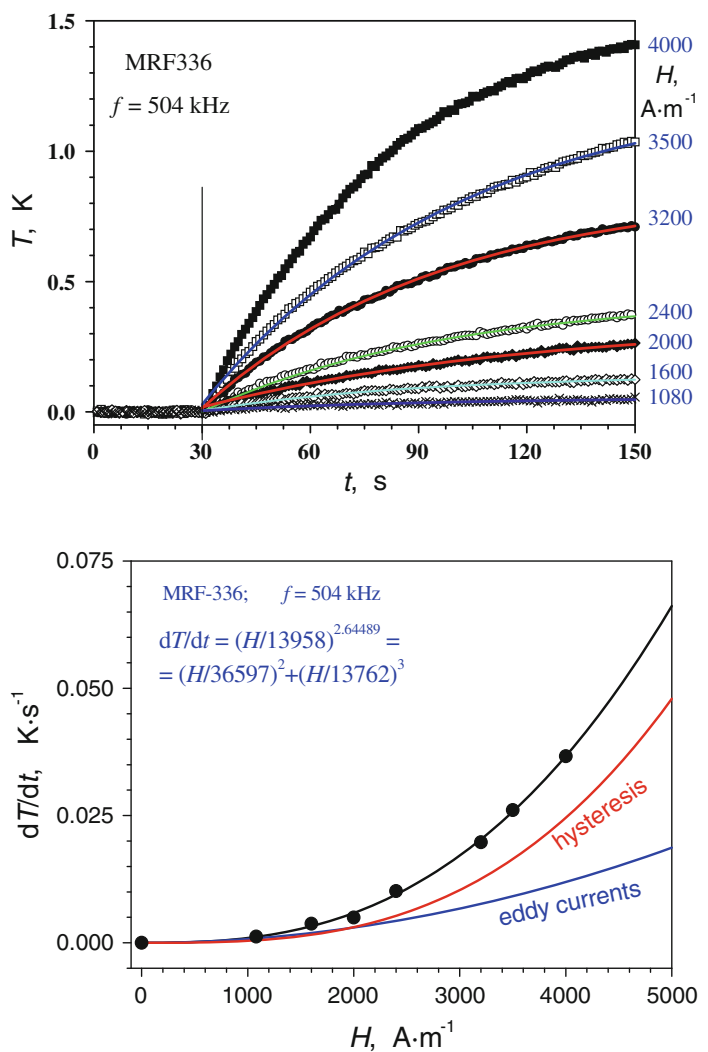

The dependence of $(\mathrm{d} T / \mathrm{d} t)_{t=0}$ on the alternating magnetic field strength amplitude $H$ at a frequency $f=504 \mathrm{kHz}$ for the MR sample is presented in Fig. 5.

For the experimental data $(\bullet)$, the function was fitted in the form [11],

$$
\left(\frac{\mathrm{d} T}{\mathrm{~d} t}\right)_{t=0}=\left(\frac{H}{a}\right)^{n},
$$

where $a$ and $n$ are the parameters obtained from the fit of the exponential function to the experimental data. For the case when only ferromagnetic particles (multidomains) are used in the experiment, the value of the exponent (index) ought to be three. Because in our experiment $n \cong 2.64$, we can suppose that eddy currents are also the reason for that. So in that case, we can write an expression which includes both sources of released heat energy.

Taking into account additively the principle of energy, we may write that the released power of losses proportional to $(\mathrm{d} T / \mathrm{d} t)_{t=0}$ consists of two components:

$$
\left(\frac{\mathrm{d} T}{\mathrm{~d} t}\right)_{t=0}=\left(\frac{H}{a}\right)^{n}=\left(\frac{H}{e}\right)^{2}+\left(\frac{H}{h}\right)^{3},
$$


Fig. 6 Contribution of eddy currents and hysteresis processes to the total release of thermal power for the MRF-336AG sample as a function of magnetic field amplitude

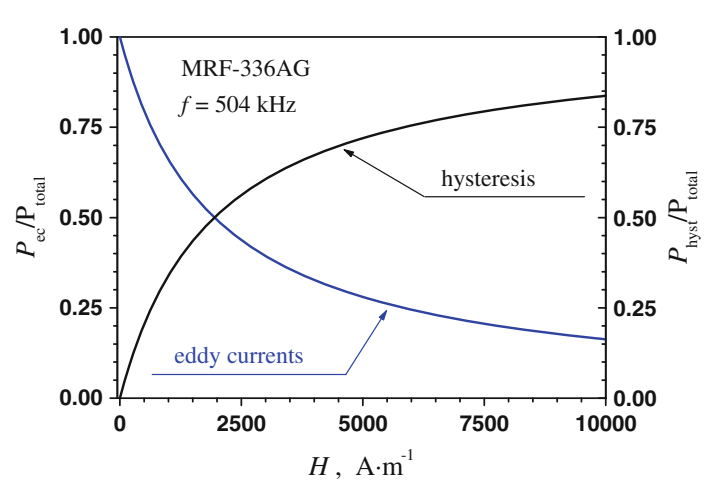

where $e$ and $h$ are parameters from the fit which are adequate in describing losses for the eddy currents and hysteresis phenomena.

After the fitting procedure, we can write the previous expression in the following way:

$$
\left(\frac{\mathrm{d} T}{\mathrm{~d} t}\right)_{t=0}=\left(\frac{H}{13958}\right)^{2.645}=\left(\frac{H}{36597}\right)^{2}+\left(\frac{H}{13762}\right)^{3} .
$$

In Fig. 6, the contribution of eddy currents and hysteresis processes to the total release of thermal energy for the MRF-336AG sample as a function of the magnetic field amplitude was presented. At a magnetic field intensity $H=1946 \mathrm{~A} \cdot \mathrm{m}^{-1}$ for the tested sample, both processes of eddy currents and hysteresis have equal participation to the total release of thermal energy.

Calorimetric measurements performed with MR fluids containing iron multidomain particles subjected to alternating magnetic fields of several intensities and frequency $f=504 \mathrm{kHz}$ allow calculation of the initial linear rise at temperature $(\mathrm{d} T / \mathrm{d} t)_{t=0}$. This quantity allows one to determine the specific absorption rate $(S A R)$ which refers to the mass unit of the sample and can be calculated from the relation,

$$
S A R\left[\frac{W}{g_{\text {sample }}}\right]=C_{P}\left(\frac{\mathrm{d} T}{\mathrm{~d} t}\right)_{t=0}=C_{P}\left[\left(\frac{H}{e}\right)^{2}+\left(\frac{H}{h}\right)^{3}\right],
$$

where $C_{p}$ is the specific heat capacity of the sample.

With the growth of the magnetic field intensity amplitude, the participation of losses connected with magnetic hysteresis is increased. $S A R$ is referenced to the mass unit of the MRF sample at the amplitude of the magnetic field strength $H=4 \mathrm{kA} \cdot \mathrm{m}^{-1}$ which equals $24.9 \mathrm{~W} \cdot \mathrm{kg}^{-1}$ at room temperature.

In our case, we may express the function in the following numerical form:

$$
S A R=C_{P}\left(\frac{\mathrm{d} T}{\mathrm{~d} t}\right)_{t=0}=0.68\left[\left(\frac{H}{36597}\right)^{2}+\left(\frac{H}{13762}\right)^{3}\right]
$$


Fig. $7 S A R$ referred to mass units of the MR sample versus magnetic field strength

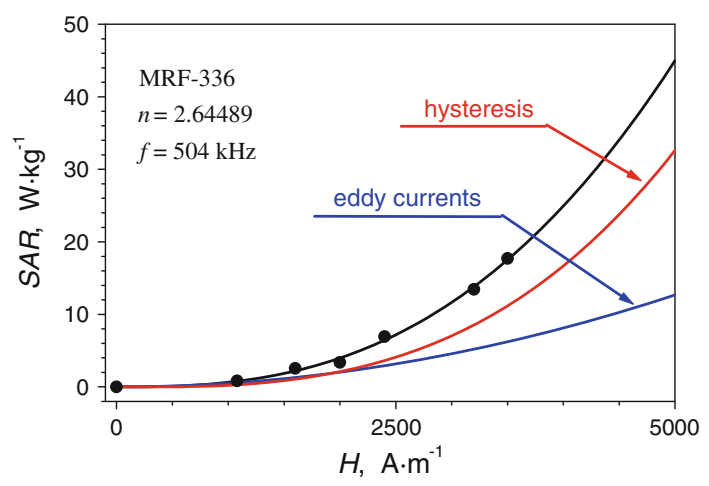

Table 1 Calculated parameters $\mathrm{d} T / \mathrm{d} t$ and $S A R$ referenced to the sample with its components and theoretical values of the effective loss power $\left\langle P_{\mathrm{O}}\right\rangle$ in mass units for some selected values of magnetic strength amplitude

\begin{tabular}{lccccc}
\hline$H\left(\mathrm{~A} \cdot \mathrm{m}^{-1}\right)$ & $\begin{array}{l}\mathrm{d} T / \mathrm{d} t \\
\left(\mathrm{~K} \cdot \mathrm{s}^{-1}\right)\end{array}$ & $\begin{array}{l}S A R \\
\left(\mathrm{~W} \cdot \mathrm{kg}^{-1}\right)\end{array}$ & $\begin{array}{l}S A R_{\mathrm{EC}} \\
\left(\mathrm{W} \cdot \mathrm{kg}^{-1}\right)\end{array}$ & $\begin{array}{l}S A R_{\text {hyst }} \\
\left(\mathrm{W} \cdot \mathrm{kg}^{-1}\right)\end{array}$ & $\begin{array}{l}\left\langle P_{\mathrm{o}}\right\rangle \text { (theory) } \\
\left(\mathrm{W} \cdot \mathrm{kg}^{-1}\right)\end{array}$ \\
\hline 1000 & 0.937 & 0.64 & 0.51 & 0.26 & 0.0018 \\
2000 & 5.863 & 3.99 & 2.03 & 2.09 & 0.0074 \\
3000 & 17.135 & 11.65 & 4.57 & 7.04 & 0.0165 \\
4000 & 36.671 & 24.94 & 8.12 & 16.70 & 0.0294 \\
5000 by extrapolation & 66.167 & 44.99 & 12.69 & 32.61 & 0.0459 \\
\hline
\end{tabular}

which is presented in Fig. 7 together with its components. The $S A R$ defined as the thermal power dissipation divided by the mass of a magnetic material can be expressed as

$$
S A R=\frac{C_{P} \rho_{\mathrm{S}}}{m_{\mathrm{Fe}}}\left(\frac{\mathrm{d} T}{\mathrm{~d} t}\right)_{t=0},
$$

where $C_{p}=680 \mathrm{~J} \cdot \mathrm{kg}^{-1} \cdot \mathrm{K}^{-1}$ is the specific heat capacity of the sample, $\rho_{\mathrm{s}}=$ $3446 \mathrm{~kg} \cdot \mathrm{m}^{-3}$ is the MRF density, $\rho_{\mathrm{Fe}}=7870 \mathrm{kgm}^{-3}$ is the iron density, $\phi_{\mathrm{v}}=0.367$ and $\phi_{\mathrm{m}}=0.8202$ are, respectively, the volume and mass concentration of iron, and $m_{\mathrm{Fe}}=2888 \mathrm{~kg} \cdot \mathrm{m}^{-3}$ is the mass of the magnetic material in the $1 \mathrm{~m}^{3}$ sample. The following values of $C_{p}, \rho_{\mathrm{s}}, \phi_{\mathrm{v}}$, and $\phi_{\mathrm{m}}$ were provided by MRF producers. In Table 1 , values which were calculated for some selected values of the magnetic strength amplitude are presented.

The theoretical dependence of the effective power loss $\left\langle P_{\mathrm{o}}\right\rangle$ referenced to a mass unit of the sample (as a function of magnetic field intensity) is shown in Fig. 8.

For the following parameter values $\langle d\rangle=2.495 \mu \mathrm{m}, f=504 \mathrm{kHz}, \rho=9.6 \times$ $10^{-8} \Omega \cdot \mathrm{m}$, and $\rho_{\mathrm{Fe}}=7870 \mathrm{~kg} \cdot \mathrm{m}^{-3}$, we obtained at $H=4 \mathrm{kA} \cdot \mathrm{m}^{-1}$ the theoretical power loss value $\left\langle P_{\mathrm{o}}\right\rangle \cong 0.0294 \mathrm{~W} \cdot \mathrm{kg}^{-1}$. 
Fig. 8 Theoretical dependence of power $\left\langle P_{\mathrm{o}}\right\rangle$ on the magnetic field strength amplitude for iron grains at frequency $f=504 \mathrm{kHz}$

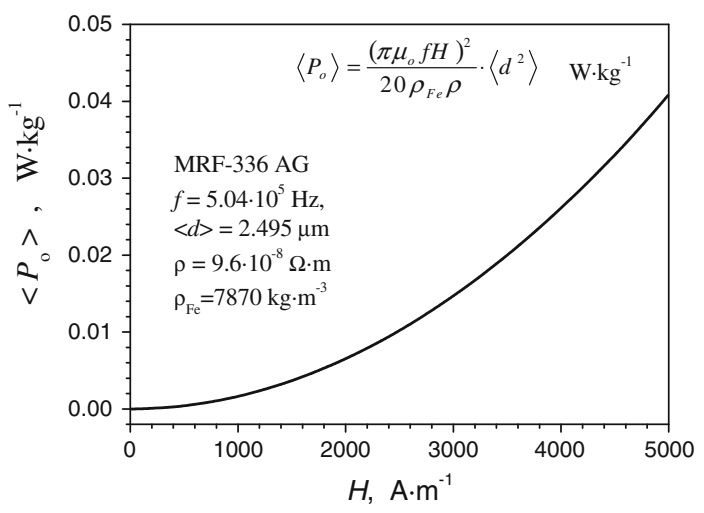

In turn, $S A R_{\mathrm{EC}}$ from our experimental results (referred only to a component caused by eddy currents) for $H=4 \mathrm{kA} \cdot \mathrm{m}^{-1}$ is equal to

$$
S A R_{\mathrm{EC}}=0.68\left(\frac{H}{36597}\right)^{2}=8.12 \mathrm{~W} \cdot \mathrm{kg}^{-1}
$$

Astonishingly, the theoretical value of the heat power $\left(\left\langle P_{\mathrm{o}}\right\rangle \cong 0.0294 \mathrm{~W} \cdot \mathrm{kg}^{-1}\right.$, at $\left.H=4 \mathrm{kA} \cdot \mathrm{m}^{-1}\right)$ is smaller than the value obtained from the experiment $\left(S A R_{\mathrm{EC}}=\right.$ $8.12 \mathrm{~W} \cdot \mathrm{kg}^{-1}$ and $S A R_{\text {total }}=24.94 \mathrm{~W} \cdot \mathrm{kg}^{-1}$ ). It is possible that in real conditions the electrical current flows in a longer circuit than in one iron grain [12]. Deriving a formula (Eq. 24), it was assumed that the induced current in an iron grain flows only in one grain. This condition may not be fulfilled in a real experiment. Moreover, the shape of the magnetic hysteresis is quite different for RF ranges than the one measured at static conditions.

\section{Conclusions}

$H^{n}$ denotes a law-type dependence of $S A R$ (where $n=2.645$ is the power) at the amplitude of the magnetic field that demonstrates the heat energy losses in the sample were generated by eddy currents and hysteresis mechanisms.

Both processes of eddy currents and hysteresis to the total release of thermal energy have equal participation at the magnetic field intensity $H=1946 \mathrm{~A} \cdot \mathrm{m}^{-1}$ for the tested sample.

The hysteresis mechanism of releasing heat energy dominates for a larger magnetic field strength amplitude $\left(H>1946 \mathrm{~A} \cdot \mathrm{m}^{-1}\right)$.

The experimental value of the heat power is larger than the value obtained from theory which may mean that the diameter of the loop with an electrical current includes some neighboring iron particles.

Acknowledgments This work was supported by the Slovak Academy of Sciences, in the framework of CEXNANOFLUID, Projects VEGA 0077 and 0043, APVV 0171-10 and Ministry of Education Agency for structural funds of EU in frame of projects Nos. 26220120021, 26220120033, and 26220120046 and by 
Polish National Science Centre grant 2011/03/B/ST7/00194 and also by POKL project: "Proinnowacyjne Kształcenie, Kompetentna Kadra, Absolwenci Przyszłości." The authors would like to thank Prof. Tomasz Hornowski for fruitful discussion.

Open Access This article is distributed under the terms of the Creative Commons Attribution License which permits any use, distribution, and reproduction in any medium, provided the original author(s) and the source are credited.

\section{Appendix: Evaluation of Energy Heat Losses in the Sphere with Radius R Placed in AC Magnetic Field as a Result of Eddy Currents}

Let us consider the single spherical iron multidomain particle (Fig. 9) which is placed in an AC magnetic field with instantaneous values of intensity,

$$
h(t)=H \sin (\omega t) .
$$

For a layer circle with thickness $\mathrm{d} r$ in a plane perpendicular to the line of magnetic field $H$, radius $A$ is equal to

$$
A=\sqrt{R^{2}-r^{2}}
$$

Hence, the magnetic flux in the layer limited by a circle with radius $h$ is

$$
\Phi_{\mathrm{h}}=\pi h^{2} \mu_{\mathrm{o}} H \sin (\omega t) .
$$

From Faraday's law, it results that the instantaneous electric voltage induced in a conductive circle layer with radius $h$ is equal to

$$
u_{\mathrm{h}}=\frac{\mathrm{d} \Phi_{\mathrm{h}}}{\mathrm{d} t}=\pi \omega h^{2} \mu_{\mathrm{o}} H \cos (\omega t) .
$$

We can say that the electrical field strength exists in this perimeter:

$$
e_{\mathrm{h}}=\frac{u_{\mathrm{h}}}{2 \pi h}=\frac{\omega h \mu_{\mathrm{o}} H \cos (\omega t)}{2}=\pi f h \mu_{\mathrm{o}} H \cos (\omega t) .
$$

Fig. 9 Auxiliary illustration for calculation of the effective loss power density in the sphere as a result of eddy currents under the influence of AC magnetic field
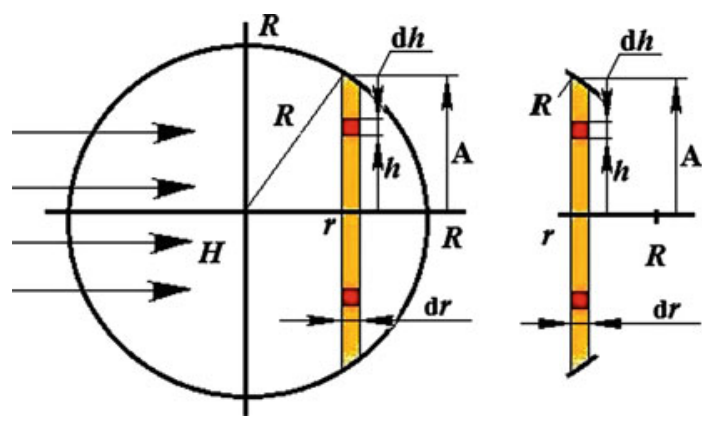
Therefore, the effective electrical field strength is

$$
\left\langle E_{\mathrm{h}}\right\rangle=\frac{E_{\max }}{\sqrt{2}}=\frac{\pi f h \mu_{\mathrm{o}} H}{\sqrt{2}} .
$$

The flow of an electric current with an effective density $\left\langle J_{\mathrm{h}}\right\rangle$ is caused by such an electrical field strength:

$$
\left\langle J_{\mathrm{h}}\right\rangle=\frac{J_{\max }}{\sqrt{2}}=\frac{\left\langle E_{\mathrm{h}}\right\rangle}{\rho}=\frac{\pi f h \mu_{\mathrm{o}} H}{\sqrt{2} \rho},
$$

where $\rho=9.6 \times 10^{-8} \Omega \cdot \mathrm{m}$ is the iron electrical resistivity.

The effective power density in the torus layer with radius $h$ is equal to

$$
\left\langle P_{\mathrm{h}}\right\rangle=\left\langle E_{\mathrm{h}}\right\rangle\left\langle J_{\mathrm{h}}\right\rangle=\frac{\left(\pi f h \mu_{\mathrm{o}} H\right)^{2}}{2 \rho} .
$$

The effective power calculated in the whole circle layer with radius $A=\left(R^{2}-r^{2}\right)^{0.5}$ and thickness $\mathrm{d} r$ is

$$
\left\langle P_{A}\right\rangle=\int_{0}^{V} P(h, r) \mathrm{d} V=\int_{0}^{h=A} \frac{\left(\pi f h \mu_{\mathrm{o}} H\right)^{2}}{2 \rho} 2 \pi \mathrm{d} r \mathrm{~d} h,
$$

where $V=2 \pi A \mathrm{~d} r$ is the volume of the disk.

$$
\begin{aligned}
& \left\langle P_{A}\right\rangle=\frac{\left(\pi f \mu_{\mathrm{o}} H\right)^{2} \pi \mathrm{d} r}{\rho} \int_{0}^{h=A} h^{3} \mathrm{~d} h=\left.\frac{\pi^{3}\left(f \mu_{\mathrm{o}} H\right)^{2}}{\rho} \mathrm{d} r \frac{h^{4}}{4}\right|_{0} ^{A} \\
& \left\langle P_{A}\right\rangle=\frac{\pi^{3}\left(f \mu_{\mathrm{o}} H\right)^{2}}{4 \rho} \mathrm{d} r\left(R^{2}-r^{2}\right)^{2} .
\end{aligned}
$$

The effective power calculated in the whole sphere is

$$
\begin{aligned}
& \langle P\rangle=2 \int_{r=0}^{r=R} \frac{\pi^{3}\left(f \mu_{\mathrm{o}} H\right)^{2}}{4 \rho}\left(R^{2}-r^{2}\right)^{2} \mathrm{~d} r=\frac{\pi^{3}\left(f \mu_{\mathrm{o}} H\right)^{2}}{2 \rho} \int_{0}^{R}\left(R^{2}-r^{2}\right)^{2} \mathrm{~d} r \\
& =\int_{0}^{R}\left(R^{2}-r^{2}\right)^{2} \mathrm{~d} r=\int_{0}^{R}\left(R^{4}-2 R^{2} r^{2}+r^{4}\right) \mathrm{d} r \\
& =\left|R^{4} r-2 R^{2} \frac{r^{3}}{3}+\frac{r^{5}}{5}\right|_{0}^{R}=R^{5}-2 \frac{R^{5}}{3}+\frac{R^{5}}{5}=\frac{8 R^{5}}{15}
\end{aligned}
$$


Therefore, the final effective power calculated in the whole sphere is

$$
\langle P\rangle=\frac{\pi^{3}\left(f \mu_{\mathrm{o}} H\right)^{2}}{2 \rho} \frac{8 R^{5}}{15}=\frac{4 \pi^{3} R^{5}\left(f \mu_{\mathrm{o}} H\right)^{2}}{15 \rho} .
$$

Taking into account the volume $V=4 \pi R^{3} / 3$, the effective power density in the sphere is equal to

$$
\frac{\langle P\rangle}{V}=\frac{3 \cdot 4 \pi^{3} R^{5}\left(f \mu_{\mathrm{o}} H\right)^{2}}{15 \rho 4 \pi R^{3}}=\frac{\left(\pi R f \mu_{\mathrm{o}} H\right)^{2}}{5 \rho} .
$$

In turn, a power $P_{\mathrm{o}}$ in mass units of the iron sphere is equal to

$$
\begin{aligned}
P_{\mathrm{o}}\left[\frac{\mathrm{W}}{\mathrm{kg}}\right] & =\frac{\langle P\rangle}{V \rho_{\mathrm{Fe}}}=\frac{3}{4 \pi R^{3} \rho_{\mathrm{Fe}}} \frac{4 \pi^{3} R^{5}\left(f \mu_{\mathrm{o}} H\right)^{2}}{15 \rho}=\frac{\left(\pi \mu_{\mathrm{o}} R f H\right)^{2}}{5 \rho_{\mathrm{Fe}} \rho} \\
P_{\mathrm{o}} & =\frac{\left(\pi \mu_{\mathrm{o}} R f H\right)^{2}}{5 \rho_{\mathrm{Fe}} \rho} .
\end{aligned}
$$

For a polydispersion system of magnetic grains where the mean square of the grain diameter is $\left\langle d^{2}\right\rangle$, the effective loss power $P_{\mathrm{o}}$ released in mass units is equal to

$$
\left\langle P_{\mathrm{o}}\right\rangle=\frac{\left(\pi \mu_{\mathrm{o}} f H\right)^{2}}{20 \rho_{\mathrm{Fe}} \rho}\left\langle d^{2}\right\rangle .
$$

\section{References}

1. S. Taketomi, Jordan J. Phys. 4, 1 (2011)

2. M. Ma, Y. Wu, J. Zhou, Y. Sun, Y. Zhang, N. Gu, J. Magn. Magn. Mater. 268, 33 (2004)

3. D.C. Jiles, D.L. Atherton, J. Magn. Magn. Mater. 61, 48 (1986)

4. G. Herzer, IEEE Trans. Mag. 26, 1397 (1990)

5. R. Hergt, R. Hiergeist, M. Zeisberger, G. Glöckl, W. Weitschies, L.P. Ramirez, I. Hilger, W.A. Kaiser, J. Magn. Magn. Mater. 280, 358 (2004)

6. R. Hergt, W. Andrä, C.G. d'Ambly, I. Hilger, W.A. Kaiser, U. Richter, H.G. Schmidt, IEEE Trans. Mag. 34, 3745 (1998)

7. R. Hiergeist, W. Andrä, N. Buske, R. Hergt, I. Hilger, U. Richter, W.A. Kaiser, J. Magn. Magn. Mater. 201, 420 (1999)

8. L. Rayleigh, Philos. Mag. 23, 255 (1887)

9. R.M. Bozorth, Ferromagnetism (IEEE Press, New York, 1978)

10. A. Skumiel, A. Józefczak, M. Timko, P. Kopčanský, F. Herchl, M. Koneracká, N. Tomašovičová, Int. J. Thermophys. 28, 1461 (2007)

11. A. Skumiel, M. Izydorzak, M. Leonowicz, A.D. Pomogailo, G.I. Dzhardimalieva, Int. J. Thermophys. 32, 1973 (2011)

12. I. Bica, J. Magn. Magn. Mater. 299, 412 (2006) 